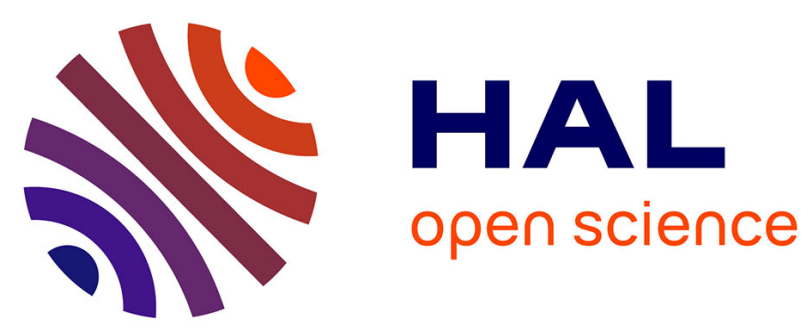

\title{
Interface collisions
}

F. D. A. Aarão Reis, Olivier Pierre-Louis

\section{- To cite this version:}

F. D. A. Aarão Reis, Olivier Pierre-Louis. Interface collisions. Physical Review E , 2018, 97 (4), pp.040801. 10.1103/PhysRevE.97.040801 . hal-02289853

\section{HAL Id: hal-02289853 \\ https://univ-lyon1.hal.science/hal-02289853}

Submitted on 11 Feb 2021

HAL is a multi-disciplinary open access archive for the deposit and dissemination of scientific research documents, whether they are published or not. The documents may come from teaching and research institutions in France or abroad, or from public or private research centers.
L'archive ouverte pluridisciplinaire HAL, est destinée au dépôt et à la diffusion de documents scientifiques de niveau recherche, publiés ou non, émanant des établissements d'enseignement et de recherche français ou étrangers, des laboratoires publics ou privés. 


\title{
Interface collisions
}

\author{
F. D. A. Aarão Reis ${ }^{1}$ and O. Pierre-Louis ${ }^{2}$ \\ ${ }^{1}$ Instituto de Física, Universidade Federal Fluminense, Avenida Litorânea s/n, 24210-340 Niterói RJ, Brazil \\ ${ }^{2}$ Institut Lumière Matière, UMR5306 Université Lyon 1-CNRS, Université de Lyon 69622 Villeurbanne, France
}

(Received 10 October 2017; published 9 April 2018)

\begin{abstract}
We provide a theoretical framework to analyze the properties of frontal collisions of two growing interfaces considering different short-range interactions between them. Due to their roughness, the collision events spread in time and form rough domain boundaries, which defines collision interfaces in time and space. We show that statistical properties of such interfaces depend on the kinetics of the growing interfaces before collision, but are independent of the details of their interaction and of their fluctuations during the collision. Those properties exhibit dynamic scaling with exponents related to the growth kinetics, but their distributions may be nonuniversal. Our results are supported by simulations of lattice models with irreversible dynamics and local interactions. Relations to first passage processes are discussed and a possible application to grain-boundary formation in two-dimensional materials is suggested.
\end{abstract}

DOI: 10.1103/PhysRevE.97.040801

Interface motion and collisions are ubiquitous in nonequilibrium systems. For example, in graphene growth on metal substrates, monocrystalline domains grow and meet, ultimately forming a polycrystalline film with grain boundaries [1-4] that influence material properties $[5,6]$. The formation of rough domain boundaries via interface collisions is encountered in many other systems undergoing domain growth, such as bacterial colonies [7]. Motivated by the selection of grains in crystal growth [8] or of species in population dynamics [9], domain boundary formation has been investigated within competitive growth models, where two interfaces grow in the same direction generating two types of domains growing side by side. The domain boundary exhibits a self-similar behavior [8], which can be affected by the average orientation of the growing interfaces [10]. However, fewer studies have considered domain boundary formation by frontal collisions, where colliding interfaces are parallel in average. Based on simulations of the Eden model, Albano et al. $[11,12]$ have exhibited numerical evidence suggesting dynamic scaling.

Furthermore, interface collisions do not always produce a domain boundary, and instead interfaces may simply annihilate. In such cases, the collision spreads in time due to the roughness of the growing fronts. This is, for example, observed in magnetic domains [13], reaction fronts [14], turbulent liquid crystals [15], burning paper [16], forest fires [17], and layer by layer crystal growth [18].

In this Rapid Communication, we determine both the roughness of the resulting domain boundary, and the spreading of the collision in time during frontal collisions. We use several different models of interface growth with irreversible rules and short-range interactions between the two interfaces. We show that the distribution and spatial correlations of collision times and of the resulting domain boundary are independent of the details of the interactions between the two interfaces, and only depend on the roughness that builds up before collision. Dynamic scaling appears as a consequence of these results.
The asymptotic distributions are dictated by the interface with the largest roughness when the growth exponent of the two colliding interfaces are different, and those distributions are nonuniversal when the growth exponents are equal.

We performed simulations using well-known onedimensional irreversible lattice growth models: random deposition (RD) with a sticking coefficient [19], a modified Family model [20], and restricted solid on solid models [21] with maximum height differences 1 (RSOS) or 2 (RSOS2). Their rules are described in Fig. 1(a). The lattice constant is the unit length and the interface length is denoted as $L$. The unit time is set by $L$ attempts of particle deposition; rejection of such attempts are possible in RSOS and RSOS2 models or after collision events with short-range interactions (defined below).

We denote the two interface positions at time $t$ and abscissa $x$ as $h_{-}(x, t)$ and $h_{+}(x, t)$. They are initially flat and located at positions $h_{ \pm}(x, t=0)= \pm d_{0}$. During growth, these interfaces move toward each other and collide. At each $x$ the collision time $t_{c}(x)$ and the locus of the collision $h_{c}(x)$ obey

$$
h_{+}\left[x, t_{c}(x)\right]=h_{-}\left[x, t_{c}(x)\right]=h_{c}(x) .
$$

Since we consider irreversible growth models, interfaces only move forward and, consequently, they only pass one time at a given height. Thus, $t_{c}(x)$ and $h_{c}(x)$ are uniquely defined by Eq. (1). Collisions are studied when both interfaces are in their growth regimes, i.e., with time increasing roughness [19,22].

The growth models are supplemented with rules describing the interaction of the interfaces as they collide. The first rule, which is illustrated in Fig. 1(b), accounts in a simple way for short-range interactions: the interfaces stop growing at each column $x$ when they meet, i.e., when Eq. (1) is satisfied. Since particle deposition depends on the height of neighboring sites (except in RD), the collision at a given column affects the subsequent growth of its neighbors. An example of the dynamics with short-range interaction is presented in Fig. 1(d). The second rule considers noninteracting interfaces which 


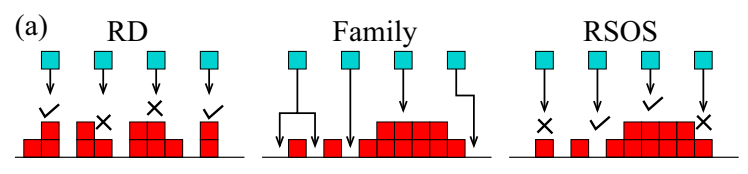

(b)

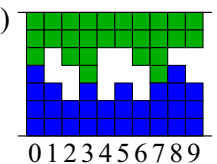

(c)

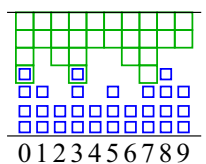

(d)

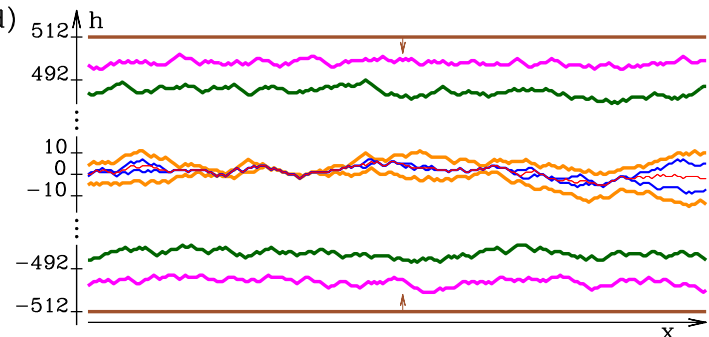

FIG. 1. Interface collision models. (a) Growth models. Random deposition (RD) model: at each time step, an incident particle sticks with probability $p$ in each column. Modified Family model: the incident particle aggregates at the column of incidence if no nearest-neighbor (NN) column has smaller height; if only one NN has a smaller height, it aggregates at that column, and if two NN columns have smaller heights, one of them is randomly chosen. RSOS models: the particle sticks only if the resulting differences of heights between all NN columns do not exceed 1 (RSOS model) or 2 (RSOS2 model). (b) Schematics of collision with short-range interaction, with growth ceasing at columns 0,3 , and 7. (c) Phantom collision, where growth continued in columns 0 (advance of upper interface) and 3 (advance of lower interface). (d) Collision simulation $\left(d_{0}=2^{9}\right)$ with two interfaces growing with the RSOS model in opposite directions and colliding with short-range interaction.

continue to grow as if the opposite interface was not there. This rule, hereafter denoted as phantom collision, is illustrated in Fig. 1(c) (movies of collisions with both types of rules are reported as Supplemental Material [23]).

We assume that interfaces move with constant and modeldependent average velocities $v_{ \pm}$[24]. We have $v_{ \pm}=p_{ \pm}$in RD, and $v=1$ in the Family model by construction. Moreover, we extracted from simulations $v=0.41904(10)$ for RSOS, and $v=0.6036(3)$ for RSOS2. The relative velocity of the two interfaces is $\bar{v}=v_{-}+v_{+}$, leading to the average collision time $t_{0}=2 d_{0} / \bar{v}$, while the average position of the collision is $h_{0}=d_{0}\left(v_{-}-v_{+}\right) / \bar{v}$. The deviations of $t_{c}(x)$ and $h_{c}(x)$ from these average values are denoted as

$$
\begin{gathered}
\delta t_{c}(x)=t_{c}(x)-t_{0}, \\
\delta h_{c}(x)=h_{c}(x)-h_{0} .
\end{gathered}
$$

We then define the distributions $F_{c}\left(\delta t_{c}\right)$ of collision times, and $P_{c}\left(\delta h_{c}\right)$ of collision loci for an initial distance $2 d_{0}$.

The first striking point revealed by simulations is the irrelevance of short-range interactions on the statistical properties of the collisions. Indeed, for $d_{0}$ large enough, the distributions $F_{c}\left(\delta t_{c}\right)$ and $P_{c}\left(\delta h_{c}\right)$ in phantom collisions are found to be identical to those with short-range interactions. This is shown in Fig. 2 for collision between interfaces governed by identical or different models.

This result suggests that interactions during collision are irrelevant. We thus define the distributions $P_{ \pm}\left(\zeta_{ \pm} ; t\right)$ of interface fluctuations $\zeta_{ \pm}(x, t)=\mp\left[h_{ \pm}(x, t)-\left\langle h_{ \pm}(x, t)\right\rangle\right]$ in absence of collision (with this definition $\zeta>0$ for fluctuations in the direction of growth). Assuming that interactions are irrelevant, we replace interface fluctuations by $\zeta_{ \pm}$, and rewrite Eq. (2) using Eq. (1):

$$
\begin{aligned}
\delta t_{c}(x) & =-\frac{\zeta_{+}\left[x, t_{0}+\delta t_{c}(x)\right]+\zeta_{-}\left[x, t_{0}+\delta t_{c}(x)\right]}{\bar{v}} \\
\delta h_{c}(x) & =\frac{-v_{-} \zeta_{+}\left[x, t_{0}+\delta t_{c}(x)\right]+v_{+} \zeta_{-}\left[x, t_{0}+\delta t_{c}(x)\right]}{\bar{v}}
\end{aligned}
$$

For large $t_{0}$, we expect $\delta t_{c}(x) \ll t_{0}$, and hence to leading order we approximate $t_{0}+\delta t_{c}(x)$ by $t_{0}$ in the right-hand side of Eqs. (3). We therefore define

$$
\begin{aligned}
\delta t_{c}^{0}(x) & =-\frac{\zeta_{+}\left(x, t_{0}\right)+\zeta_{-}\left(x, t_{0}\right)}{\bar{v}} \\
\delta h_{c}^{0}(x) & =\frac{-v_{-} \zeta_{+}\left(x, t_{0}\right)+v_{+} \zeta_{-}\left(x, t_{0}\right)}{\bar{v}} .
\end{aligned}
$$

These quantities can be obtained as follows: (i) perform the evolution as if interfaces could evolve and freely cross without interacting up to time $t_{0}$; (ii) freeze the interfaces at $t=t_{0}$ and slide them (forward and backward in time) without shape change and with their own average velocity $v_{ \pm}$; (iii) measure the collision times $t_{c}^{0}(x)$ and locations $h_{c}^{0}(x)$. This process, hereafter referred to as the freeze-and-slide approximation, corresponds to a situation where fluctuations during collision are absent.

Since $\zeta_{+}$and $\zeta_{-}$are independent, the probability distributions resulting from Eq. (4) read

$$
\begin{aligned}
F_{c}\left(\delta t_{c}\right) & =\bar{v} \int d \zeta_{+} P_{+}\left(\zeta_{+} ; t_{0}\right) P_{-}\left(-\delta t_{c} \bar{v}-\zeta_{+} ; t_{0}\right) \\
P_{c}\left(\delta h_{c}\right) & =\frac{\bar{v}}{v_{+}} \int d \zeta_{+} P_{+}\left(\zeta_{+} ; t_{0}\right) P_{-}\left(\frac{-\delta h_{c} \bar{v}+\zeta_{+} v_{-}}{v_{+}} ; t_{0}\right)
\end{aligned}
$$

Using $P_{ \pm}\left(\zeta_{ \pm}, t_{0}\right)$ obtained numerically from simulations of interfaces without collision, we calculated these convoluted distributions for collisions with five pairs of models, as shown in Fig. 2. In all cases, there is excellent agreement with distributions obtained in collision simulations, confirming the validity of the freeze-and-slide approximation.

Based on this result, we now show that collision properties obey simple scaling laws. From dynamic scaling [19,25], time correlation functions of a growing interface are characterized by the growth exponent $\beta$ :

$$
\left\langle[\zeta(x, t+\tau)-\zeta(x, t)]^{2}\right\rangle=B|\tau|^{2 \beta},
$$

as long as the correlation length $\xi_{\text {corr }} \sim t^{\beta / \alpha}$ is smaller than the interface length $L$. The roughness exponent $\alpha$ characterizes spatial correlations at short enough distances $\xi \ll \xi_{\text {corr }}[19,22]$ 


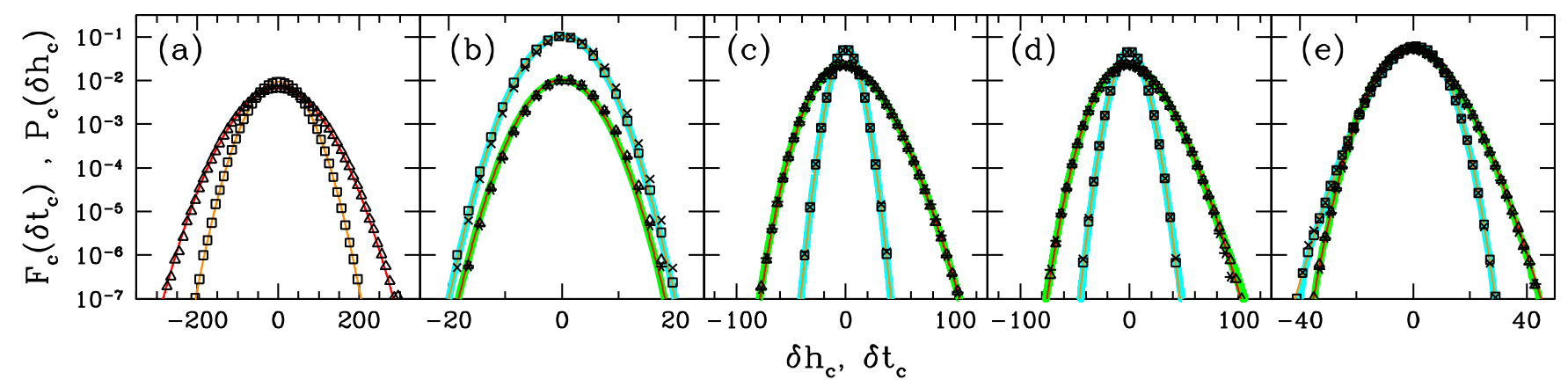

FIG. 2. Distributions of collision times and loci. Models: (a) RD-RD with $p_{+}=0.5$ and $p_{-}=1$, (b) Family-Family, (c) RSOS-RSOS, (d) RSOS-RSOS2, (e) RSOS-Family. Symbols indicate simulation results with $d_{0}=2^{12}$ and $L=2^{32}$, and with short-range interaction [ $\square$ : $\left.P_{c}\left(\delta h_{c}\right), \triangle: F_{c}\left(\delta t_{c}\right)\right]$ or phantom collision $\left[\times: P_{c}\left(\delta h_{c}\right), *: F_{c}\left(\delta t_{c}\right)\right]$. Thick curves are obtained from Eqs. (5a) and (5b) (green, blue) using distributions from interfaces without collision calculated numerically. Thin lines are distributions obtained from Eqs. (5a) and (5b) (red, orange) using theoretical universal distributions (Gaussian or Tracy-Widom) with variances extracted from simulations of interfaces without collisions. In (b), $F_{c}\left(\delta t_{c}\right)$ is shifted down by one unit for the sake of clarity.

via

$$
\left\langle[\zeta(x+\xi, t)-\zeta(x, t)]^{2}\right\rangle=A|\xi|^{2 \alpha} .
$$

Within this description, RD corresponds to diffusive dynamics with $\beta=1 / 2$ without lateral correlation. The other models belong to universality classes with subdiffusive time correlations [19,22]: Edwards-Wilkinson (EW) class with $\beta=$ $1 / 4$ and $\alpha=1 / 2$ for the Family model; Kardar-Parisi-Zhang (KPZ) class with $\beta=1 / 3$ and $\alpha=1 / 2$ for RSOS and RSOS2.

The variances of the distributions $F_{c}\left(\delta t_{c}\right)$ and $P_{c}\left(\delta h_{c}\right)$ are obtained from Eq. (4) as

$$
\begin{aligned}
\left\langle\delta t_{c}(x)^{2}\right\rangle & =\frac{B_{+} t_{0}^{2 \beta_{+}}+B_{-} t_{0}^{2 \beta_{-}}}{\bar{v}^{2}}, \\
\left\langle\delta h_{c}(x)^{2}\right\rangle & =\frac{v_{-}^{2} B_{+} t_{0}^{2 \beta_{+}}+v_{+}^{2} B_{-} t_{0}^{2 \beta_{-}}}{\bar{v}^{2}},
\end{aligned}
$$

where we have used that $\left\langle\zeta_{ \pm}\left(x, t_{0}\right)^{2}\right\rangle=B_{ \pm} t_{0}^{2 \beta_{ \pm}}$from Eq. (6) with $\zeta_{ \pm}(x, t=0)=0$. If $\beta_{+}=\beta_{-}$, both terms in the righthand-side of Eqs. (8) are equally relevant. Otherwise, for $\beta_{+} \neq \beta_{-}$, the term with the largest exponent is asymptotically dominant, and the variances scale with exponent $2 \beta_{m}$, where $m=+$ when $\beta_{+} \geqslant \beta_{-}$and $m=-$ when $\beta_{-}>\beta_{+}$.

In collisions with the RD model, each column is equivalent to an independent first passage process, thereby providing an alternative analytical derivation of Eqs. (5) and (8) in a special case. The resulting distribution for the height $h_{ \pm}$of one column is a binomial distribution [19]. Using Stirling's formula, one obtains a Gaussian distribution for $P\left(h_{+} ; t\right)$ at long times with variance $\left\langle\zeta_{ \pm}^{2}\right\rangle=\left\langle\left(h_{ \pm}-v_{ \pm} t\right)^{2}\right\rangle=4 D_{ \pm} t$, where $v_{ \pm}=p_{ \pm}$and $D_{ \pm}=p_{ \pm}\left(1-p_{ \pm}\right) / 2$ is the diffusion constant. Comparison with Eq. (6) leads to $\beta_{ \pm}=1 / 2$ and $B_{ \pm}=2 D_{ \pm}$. In one column, the collision then reduces to the first passage process of two particles undergoing biased diffusion toward each other, which has a well-known solution [26]. Since columns are independent, the average over realizations leads to the same result as the average over the interface size $L$, providing the distributions $F_{c}\left(\delta t_{c}\right)$ and $P_{c}\left(\delta h_{c}\right)$ (detailed expressions are in the Supplemental Material). In the limit where $d_{0} \gg 1$ and $d_{0} \gg D_{ \pm} / v_{ \pm}$, one finds Gaussians in agreement with Eqs. (5), with variances given by Eqs. (8).
For collisions with other models, the estimates of the exponents of the variances of $\delta t_{c}$ and $\delta h_{c}$ were obtained in simulations and are shown in Table I (numerical procedures are in the Supplemental Material). They agree with the exponent $\beta_{m}$ expected from Eq. (8). Using the theoretically predicted value of $\beta_{m}$ and the variances from simulations, we calculated the ratios $\left\langle\delta t_{c}^{2}\right\rangle /\left(2 d_{0}\right)^{2 \beta_{m}}$ and $\left\langle\delta h_{c}^{2}\right\rangle /\left(2 d_{0}\right)^{2 \beta_{m}}$ and extrapolated them to $d_{0} \rightarrow \infty$. As shown in Table I, the results agree with the estimates obtained from Eq. (8) with the values of $v$ and $B$ extracted from simulations of interfaces in the absence of collision $[B=0.4495(10)$ for the Family model; $B=0.254$ (1) for RSOS; $B=0.552$ (2) for RSOS2].

Beyond exponents, the different universality classes impose that $P(\zeta ; t)=f(\zeta / W) / W$, with $W=B^{1 / 2} t^{\beta}$ and universal distributions $f$ at long times: Gaussian for RD and EW class, and Tracy-Widom for the KPZ class [27,28]. Inserting this ansatz into Eq. (5) and using the variances from the corresponding models without collision at $t_{0}$, we obtain distributions $F_{c}$ and $P_{c}$ in good agreement with collision simulations, as shown in Fig. 2 (this is confirmed by the analysis of the skewness

TABLE I. Comparison of exponents and amplitudes calculated in simulations with short-range interaction (upper values) and predicted by the freeze-and-slide approximation (lower values).

\begin{tabular}{lcccc}
\hline \hline+ & Family & RSOS & RSOS & RSOS \\
- & Family & RSOS & RSOS2 & Family \\
\hline$\beta\left(\delta t_{c}\right)$ & $0.248(5)$ & $0.329(4)$ & $0.333(1)$ & $0.330(15)$ \\
Eq. $(8 \mathrm{a})$ & $1 / 4$ & $1 / 3$ & $1 / 3$ & $1 / 3$ \\
$\left\langle\delta t_{c}^{2}\right\rangle /\left(2 d_{0}\right)^{2 \beta}$ & $0.159(1)$ & $0.815(2)$ & $0.761(1)$ & $0.095(25)$ \\
Eq. $(8 \mathrm{a})$ & $0.1589(4)$ & $0.814(3)$ & $0.759(4)$ & $0.0999(4)$ \\
$A_{\delta t_{c}}$ & $0.318(7)$ & $2.34(2)$ & $3.6(2)$ & $0.75(3)$ \\
Eq. $(9 \mathrm{a})$ & $0.320(5)$ & $2.35(3)$ & $3.49(7)$ & $0.728(10)$ \\
$\beta\left(\delta h_{c}\right)$ & $0.250(2)$ & $0.333(1)$ & $0.334(1)$ & $0.327(3)$ \\
Eq. $(8 \mathrm{~b})$ & $1 / 4$ & $1 / 3$ & $1 / 3$ & $1 / 3$ \\
$\left\langle\delta h_{c}^{2}\right\rangle /\left(2 d_{0}\right)^{2 \beta}$ & $0.1593(3)$ & $0.1435(5)$ & $0.179(1)$ & $0.100(5)$ \\
Eq. $(8 \mathrm{~b})$ & $0.1589(4)$ & $0.1429(6)$ & $0.1785(6)$ & $0.0999(4)$ \\
$A_{\delta h_{c}}$ & $0.320(5)$ & $0.414(6)$ & $0.745(15)$ & $0.475(20)$ \\
Eq. $(9 \mathrm{~b})$ & $0.320(5)$ & $0.413(5)$ & $0.761(14)$ & $0.466(5)$ \\
\hline \hline
\end{tabular}


and kurtosis in the Supplemental Material). If $\beta_{+} \neq \beta_{-}$, this scaling ansatz can be inserted in Eqs. (5). We then find that, to leading order, the distributions of time and locus of collision follow the universal distribution of the growing interface with exponent $\beta_{m}: F_{c}\left(\delta t_{c}\right)=f_{m}\left(-\delta t_{c} / T_{c}\right) / T_{c}$, where $T_{c}=W_{m} / \bar{v}$, and $P_{c}\left(\delta h_{c}\right)=f_{m}\left(-\delta t_{c} / W_{c}\right) / W_{c}$, where $W_{c}=v_{-m} W_{m} / \bar{v}$. In contrast, when $\beta_{+}=\beta_{-}$the distributions $P_{c}$ and $F_{c}$ resulting from Eq. (8) cannot be rescaled by a single time or length scale; they are nonuniversal in the sense that they depend on (ratios of) nonuniversal model-dependent parameters $\left(v_{ \pm}\right.$and $\left.B_{ \pm}\right)$.

We now turn to spatial correlations. Approximating $\delta t_{c}$ and $\delta h_{c}$ by Eq. (4) and using Eq. (7), we find that at distances smaller than the correlation lengths of the two interfaces, spatial correlations obey

$$
\begin{aligned}
\left\langle\left[\delta t_{c}(x+\xi)-\delta t_{c}(x)\right]^{2}\right\rangle & =\frac{A_{+}|\xi|^{2 \alpha_{+}}+A_{-}|\xi|^{2 \alpha_{-}}}{\bar{v}^{2}}, \\
\left\langle\left[\delta h_{c}(x+\xi)-\delta h_{c}(x)\right]^{2}\right\rangle & =\frac{v_{-}^{2} A_{+}|\xi|^{2 \alpha_{+}}+v_{+}^{2} A_{-}|\xi|^{2 \alpha_{-}}}{\bar{v}^{2}} .
\end{aligned}
$$

Thus, to leading order, correlations scale in $\xi$ with an exponent $\alpha_{c}=\max \left[\alpha_{+}, \alpha_{-}\right]$.

In the absence of collisions, the scaling in Eq. (7) is observed numerically in narrow ranges of $\xi$ even at long times. However, using the known values of $\alpha_{ \pm}$and an extension of the procedure developed in [29], we estimated the amplitudes $A=0.64(1)$ for the Family model, $A=0.825(10)$ for RSOS, and $A=2.82(6)$ for RSOS2. The same method is used to estimate $A_{\delta t_{c}} \equiv\left\langle\left[\delta t_{c}(x+\xi)-\delta t_{c}(x)\right]^{2}\right\rangle /|\xi|^{2 \alpha_{c}}$ and $A_{\delta h_{c}} \equiv\left\langle\left[\delta h_{c}(x+\xi)-\delta h_{c}(x)\right]^{2}\right\rangle /|\xi|^{2 \alpha_{c}}$. The results shown in Table I indicate good agreement between Eq. (9) and the simulations (the convergence to these values is presented in the Supplemental Material). For family-RSOS collisions, observe that $\alpha_{+}=\alpha_{-}$, thus EW correlations contribute to the lateral correlation of the collision interface at small length scales, although distributions $F_{c}$ and $P_{c}$ belong to the KPZ class.

In addition, dynamic scaling provides a rationale for the irrelevance of short-range interactions. Indeed, from Eqs. (8), the collision duration $T_{c}=\left\langle\delta t_{c}(x)^{2}\right\rangle^{1 / 2} \sim W_{c} / \bar{v}$, where $W_{c}=$ $\left\langle\delta h_{c}(x)^{2}\right\rangle^{1 / 2}$. Thus, during collision, lateral correlations propagate on a distance $\xi_{\text {coll }} \sim T_{c}^{\beta / \alpha} \sim W_{c}^{\beta / \alpha}$ (here the indices of $\alpha$ and $\beta$ can be + or - without affecting the conclusions). Since the distance between the interfaces during collision is $\sim W_{m} \sim W_{c}$, we expect the typical distance between contact points to be $\xi_{\text {contact }} \sim W_{c}^{1 / \alpha}$ from Eq. (9b). For normal dynamic scaling, $\beta<\alpha \leqslant 1[19,22]$, thus we have $\xi_{\text {coll }} \ll W_{c} \leqslant \xi_{\text {contact }}$ at long times. Hence, interactions influence the collisions in the vicinity of contact points, but these perturbations do not have time to propagate between contact points during the collision time. Thus, interactions are irrelevant to leading order.

Scaling also imposes the irrelevance of fluctuations during collision. Indeed, we have $T_{c} \sim W_{c} / \bar{v} \sim t_{0}^{\beta_{m}} \ll t_{0}$, justifying the separation of scales at the origin of the freeze-and-slide approximation. Furthermore, from Eqs. (3), (4), and (6), we have $\left\langle\left(\delta t_{c}-\delta t_{c}^{0}\right)^{2}\right\rangle \sim\left\langle\left[\zeta\left(t_{0}+\delta t_{c}\right)-\zeta\left(t_{0}\right)\right]^{2}\right\rangle \sim T_{c}^{2 \beta_{m}} \sim t_{0}^{2 \beta_{m}^{2}}$. Thus, $\left\langle\left(\delta t_{c}-\delta t_{c}^{0}\right)^{2}\right\rangle \ll T_{c}^{2} \sim t_{0}^{2 \beta_{m}}$. This means that deviations of $\delta t_{c}$ from $\delta t_{c}^{0}$ are negligible, i.e., fluctuations during col- lision are irrelevant. This result and a similar analysis of $\left\langle\left(\delta h_{c}-\delta h_{c}^{0}\right)^{2}\right\rangle$ are presented in the Supplemental Material. Similarly, when the growing fronts reach the late-times stationary state where the roughness saturates to a value that depends on $L$, scaling as a function of $L$ is also expected for large $L$, as observed in simulations in Refs. [11,12].

As a final remark, we conjecture that our results for irreversible growth should directly extend to growing interfaces with particle attachment and detachment, that may exhibit more than one passage obeying Eq. (1). In such cases, the predictions reported above describe the average passage time for phantom collisions instead of their first passage time. Nevertheless, the difference between the first passage time and the average passage time is dictated by the fluctuations during the collision, which were shown to be irrelevant. As a consequence, the first passage time should also be well approximated by the freeze-and-slide process and our results should be valid when backward motion of the interfaces is possible. This conclusion is corroborated by the agreement discussed above between the asymptotic behaviors of the irreversible RD model and the continuum biased random walk, which exhibits both forward and backward propagation.

In conclusion, our central result is that local interactions and interface fluctuations during the collision do not affect the asymptotic statistical properties of interface collision. As a consequence, collision properties exhibit dynamic scaling with universal exponents; however, distributions can be nonuniversal when $\beta_{+}=\beta_{-}$.

Our results may be investigated with the measurement of grain-boundary roughness of two-dimensional materials such as graphene [1-4] and $\mathrm{MoS}_{2}$ [30,31]. Assume, for example, that the radius $R$ of growing two-dimensional grains is proportional to time $t$, and $\beta$ is the growth exponent of the two grain edges before collision. From Eq. (8b), we speculate that the roughness of grain boundaries will be $W \sim t^{\beta} \sim R^{\beta}$. The relation between $W$ and $R$ should therefore allow one to determine $\beta$, providing strong constraints on the possible microscopic growth mechanisms proposed in the literature $[32,33]$.

As a promising perspective, interface collisions can be considered as a generalization of first passage processes [26,34], where particles diffuse and stick or annihilate when they meet. As opposed to particles, interfaces present intrinsic roughness, which leads to a spreading of the collision in time (some parts meet earlier than others) and in space (all parts do not meet on the same plane). Hence, advances on first passage of subdiffusive systems [34,35] and in exact solutions of kinetic roughening $[27,36,37]$ should provide tools to explore the underlying links between interface collisions and first-passage processes. Natural ramifications linked to persistence [38], large deviation [39], and extremal statistics [40] of interfaces also appear when, e.g., considering the properties of first and last contacts during interface collisions.

F.D.A.A.R. acknowledges support by $\mathrm{CNPq}$ and FAPERJ (Brazilian agencies) and is grateful for the hospitality of Université Lyon 1, where part of this work was performed. O.P.L. wishes to thank Nanoheal (EU H2020 research and innovation program under Grant Agreement No. 642976), and LOTUS (Grant No. ANR-13-BS04-0004-02). 
[1] L. Gao, J. R. Guest, and N. P. Guisinger, Nano Lett. 10, 3512 (2010).

[2] P. Y. Huang, C. S. Ruiz-Vargas, A. M. van der Zande, W. S. Whitney, M. P. Levendorf, J. W. Kevek, S. Garg, J. S. Alden, C. J. Hustedt, Y. Zhu, J. Park, P. L. McEuen, and D. A. Muller, Nature (London) 469, 389 (2011).

[3] Q. Yu, L. A. Jauregui, W. Wu, R. Colby, J. Tian, Z. Su, H. Cao, Z. Liu, D. Pandey, D. Wei, T. F. Chung, P. Peng, N. P. Guisinger, E. A. Stach, J. Bao, S.-S. Pei, and Y. P. Chen, Nat. Mater. 10, 443 (2011).

[4] B. Kiraly, E. B. Iski, A. J. Mannix, B. L. Fisher, M. C. Hersam, and N. P. Guisinger, Nat. Commun. 4, 2804 (2013).

[5] W. J. Evans, L. Hu, and P. Keblinski, Appl. Phys. Lett. 96, 203112 (2010).

[6] S. Merabia and K. Termentzidis, Phys. Rev. B 89, 054309 (2014).

[7] A. Be'er, H. P. Zhang, E.-L. Florin, S. M. Payne, E. Ben-Jacob, and H. L. Swinney, Proc. Natl. Acad. Sci. USA 106, 428 (2009).

[8] Y. Saito and H. Müller-Krumbhaar, Phys. Rev. Lett. 74, 4325 (1995).

[9] J.-T. Kuhr, M. Leisner, and E. Frey, New J. Phys. 13, 113013 (2011).

[10] B. Derrida and R. Dickman, J. Phys. A: Math. Gen. 24, L191 (1991).

[11] E. V. Albano, Phys. Rev. E 56, 7301 (1997).

[12] E. V. Albano and I. M. Irurzun, J. Phys. A: Math. Gen. 34, 9631 (2001).

[13] L. Krusin-Elbaum, T. Shibauchi, B. Argyle, L. Gignac, and D. Weller, Nature (London) 410, 444 (2001).

[14] S. Atis, A. K. Dubey, D. Salin, L. Talon, P. Le Doussal, and K. J. Wiese, Phys. Rev. Lett. 114, 234502 (2015).

[15] K. A. Takeuchi and M. Sano, Phys. Rev. Lett. 104, 230601 (2010).

[16] J. Maunuksela, M. Myllys, O.-P. Kähkönen, J. Timonen, N. Provatas, M. J. Alava, and T. Ala-Nissila, Phys. Rev. Lett. 79, 1515 (1997).

[17] N. Guisoni, E. S. Loscar, and E. V. Albano, Phys. Rev. E 83, 011125 (2011).

[18] A. Pimpinelli and J. Villain, Physics of Crystal Growth (Cambridge University Press, Cambridge, UK, 1998).

[19] A. Barabási and H. Stanley, Fractal Concepts in Surface Growth (Cambridge University Press, Cambridge, UK, 1996).

[20] F. Family, J. Phys. A: Math. Gen. 19, L441 (1986).

[21] J. M. Kim and J. M. Kosterlitz, Phys. Rev. Lett. 62, 2289 (1989).

[22] J. Krug, Adv. Phys. 46, 139 (1997).

[23] See Supplemental Material at http://link.aps.org/supplemental/ 10.1103/PhysRevE.97.040801 for movies of interface collisions and detailed discussions of (i) the distributions of first passage times and loci for two particles undergoing biased diffusion, (ii) the convergence of the effective exponent of the width of the distributions, (iii) the skewness and kurtosis of the distributions, (iv) the convergence of the amplitude of spatial correlations, and (v) the difference between $\delta h_{c}^{0}, \delta t_{c}^{0}$, and $\delta t_{c}, \delta h_{c}$.

[24] Subdominant terms in the scaling behavior are known to affect front velocities with a slowly varying function [41]. These effects are negligible for the largest $d_{0}$ studied here.

[25] T. Vicsek, Fractal Growth Phenomena (World Scientific, Singapore, 1992).

[26] S. Redner, A Guide to First-Passage Processes (Cambridge University Press, Cambridge, England, 2001).

[27] T. Sasamoto and H. Spohn, Phys. Rev. Lett. 104, 230602 (2010).

[28] C. A. Tracy and H. Widom, Commun. Math. Phys. 159, 151 (1994).

[29] A. Chame and F. D. A. A. Reis, Surf. Sci. 553, 145 (2004).

[30] L. Tao, K. Chen, Z. Chen, W. Chen, X. Gui, H. Chen, X. Li, and J.-B. Xu, ACS Appl. Mater. Interfaces 9, 12073 (2017).

[31] L. Karvonen, A. Saynatjoki, M. J. Huttunen, A. Autere, B. Amirsolaimani, S. Li, R. A. Norwood, N. Pryghambarian, H. Lipsanen, G. Eda, K. Keiu, and Z. Sun, Nat. Commun. 8, 15714 (2017).

[32] E. Loginova, N. C. Bartelt, P. J. Feibelman, and K. F. McCarty, New J. Phys. 10, 093026 (2008).

[33] P. Wu, Y. Zhang, P. Cui, Z. Li, J. Yang, and Z. Zhang, Phys. Rev. Lett. 114, 216102 (2015).

[34] R. Metzler, S. Redner, and G. Oshanin, First-Passage Phenomena and Their Applications (World Scientific, Singapore, 2014), Vol. 35.

[35] T. Guérin, N. Levernier, O. Bénichou, and R. Voituriez, Nature (London) 534, 356 (2016).

[36] P. Calabrese and P. Le Doussal, Phys. Rev. Lett. 106, 250603 (2011).

[37] J. De Nardis, P. Le Doussal, and K. A. Takeuchi, Phys. Rev. Lett. 118, 125701 (2017).

[38] A. J. Bray, S. N. Majumdar, and G. Schehr, Adv. Phys. 62, 225 (2013).

[39] B. Meerson, E. Katzav, and A. Vilenkin, Phys. Rev. Lett. 116, 070601 (2016).

[40] M. Dentz, I. Neuweiler, Y. Méheust, and D. M. Tartakovsky, Phys. Rev. E 94, 052802 (2016).

[41] S. G. Alves, T. J. Oliveira, and S. C. Ferreira, J. Stat. Mech. (2013) P05007. 